\title{
Extemporaneous compounding: Cautions, controversies and convenience
}

\author{
AK Mohiuddin \\ Assistant Professor, Dept. of Pharmacy, World University of Bangladesh, Bangladesh \\ *Corresponding Author: AK Mohiuddin \\ Email: trymohi@gmail.com
}

\begin{abstract}
Pharmacists are only knowledgeable and skilled healthcare professionals dedicated to compounding and preparing medications to meet the unique needs of patients. The safe and effective extemporaneous compounding of prescription products for patients require in special care is fundamental to the pharmacy profession. But there are much to do for secundum artem. It is not at all economical for a pharmaceutical company to marketize a product in 10 different probable doses or in 5 different dosage forms to meet the needs of the entire range of individuals receiving therapy. Although development is a continuous process, companies are customizing features to meet the majority of patient needs, but the very nature of the process cannot meet all patient needs. The risk-benefit ratio of using traditionally compounded medicines is favorable for patients who require specialized medications that are not commercially available, as they would otherwise not have access to suitable treatment. However, if an FDA-approved drug is commercially available, the use of an unapproved compounded drug confers additional risk with no commensurate benefit. Published reports of independent testing by the FDA, state agencies, and others consistently show that compounded drugs fail to meet specifications at a considerably higher rate than FDA-approved drugs. Compounded sterile preparations pose the additional risk of microbial contamination to patients. In the last 11 years, three separate meningitis outbreaks have been traced to purportedly 'sterile' steroid injections contaminated with fungus or bacteria, which were made by compounding pharmacies. The 2012 outbreak has resulted in intense scrutiny of pharmacy compounding practices and increased recognition of the need to ensure that compounding is limited to appropriate circumstances.

Purpose of The Study: The article intends to physico-chemical and economic contemplations previously compounding; elements and quality control issues; compounding support, preparing, chemical supplies, kinds of compounding (exceptionally in hospital and walking care compounding). It should help to rehearse the unpremeditated readiness of fundamental and propelled definitions including pharmacopoeial and non-pharmacopoeial plans experienced in drug store practice, together with essential documentation, marking, bundling and directing prerequisites. Alongside this, they need to contemplate the investigation of definitions and their segments and relate these to the clinical execution of medicines. This will assist them with investigating, assess and report the physical attributes of details including discharge energy and relate these to quality control and pre-formulation prerequisites; relate the utilization of quality control, quality confirmation and the standards of good assembling practice to direction of medicine creation in home and abroad.
\end{abstract}

Outline: Background; Introduction; Compounding Factors; Types of Compounding; Identifying Errors and Cause.

Keywords: Pharmacy practice, Drugs, Dosage forms, Compounding, Equipment, Pharmacopeia.

\section{Introduction}

The FDA characterizes customary pharmacy compounding as the consolidating, blending, or changing of fixings to make a tweaked medication for an individual patient because of an authorized expert's remedy. The NABP further portrays compounding as the consequence of an expert's doctor prescribed medication arrange dependent on the specialist/quiet/pharmacist relationship over the span of expert practice. Customary pharmacy compounding assumes a significant job in giving access to medications to people with one of a kind restorative needs, which can't be met with a financially accessible item. For example, a prescriber may ask for that a pharmacist aggravate a suspension for a pediatric or geriatric patient unfit to swallow a medication in its economically accessible shape. In customary pharmacy compounding, an individualized medicine is set up in line with a prescriber on a little scale. Clearly extensive scale producers have exceptionally constrained chance to redo measurements quality and dose frames for a client fragment that isn't significantly sufficiently enormous because of economies of scale idea of business. Another significant distinction is that compounding drug stores are not required to report antagonistic occasions to the FDA, while unfriendly occasion detailing is compulsory for makers of FDA- managed medications. Along these lines, unfriendly occasions related with intensified medications might be hard to recognize, especially if the influenced patients are generally dispersed in various geographic territories. Enrolled and gifted pharmacists in rehearsing their craft of compounding fills in this hole to address individualized issues. Compounding has dependably been a fundamental piece of pharmacy practice; the medications, measurements structures, and gear or systems utilized are the factors. Pharmacists have special information and aptitudes and are not copied by some other calling. Pharmacy exercises to individualize tolerant treatment incorporate compounding and clinical capacities. Either work without alternate outcomes in setting pharmacy in a defenseless position. It is essential to endow a pharmacist's aptitude to alter dose amounts, frequencies, and even dose structures to upgrade consistence. Pharmacy is a mind-boggling blend of various practices and practice locales. Never again is pharmacy just community pharmacy or hospital pharmacy. Pharmacy is differing and offers numerous open doors for those ready to glance around, discover their specialty and practice pharmacy to address the issues of their own community of patients. Most compounding pharmacists seem, by all accounts, to be intrigued and amped up for their practices. Truth be told, numerous pharmacists personally associated 
with pharmaceutical consideration have now understood the significance of giving individualized patient consideration through the planning of patient-explicit items. Compounding pharmacy isn't for everybody, except as it develops, it will give an inexorably critical number of pharmacists the energy and satisfaction of utilizing their imaginative and innovative aptitudes to take care of patient issues.

\section{Rationale of Extemporaneous Compounding}

A gauge by FDA that $1-10 \%$ of all remedies require compounding was not upheld by proof information. EP systems in clinical examinations requires 'fit-for-reason' plan structure and arrangement forms, just as organization techniques that are protected, adaptable, financially savvy, and easy to adjust by a compounding pharmacist at the clinical site. Numerous commercially accessible medications are not fabricated in reasonable measurements frames for these unique populaces, e.g., fluid details for kids and the individuals who might be not able swallow tablets. Moreover, when sedate deficiencies happen or for the treatment of uncommon sicknesses, exacerbated items have a place practically speaking. Instances of adjusted medication items incorporate topical treatments, eye drops, strong or fluid oral measurements shapes, and intravenous mixtures. Compounding likewise has a place in veterinary practice, where medicines might be changed to suit sedate ingestion, digestion, and gulping capacities of various species. The utilization of little scale aggravated medications conveys certain dangers, as they are not surveyed for item quality, soundness, or adequacy. The significance of a community, group based way to deal with patient consideration and security has been entrenched inside the wellbeing field. The necessity for elective medications and measurement structures might be dictated by the patient's general specialist who recommends the medication or other partnered wellbeing experts: a discourse pathologist who recognizes a gulping trouble, a physiotherapist applying ultrasound, which may meddle with transdermal medication conveyance, or a word related advisor distinguishing that a patient can't physically control their present medication or its bundling. This features the significance for different individuals from the medicinal services groups to have information and comprehension of exacerbated medications. Among older patients, the rate of dysphagia is apparently as high as $7 \%-13 \%$, making use of accessible oral measurements plans unfeasible or hazardous. Additionally, Canadian hospital pharmacists frequently experience patients with oral, neck, or gastric disease, which may make business strong measurement definitions difficult to utilize. In view of pharmacists' point of view, utilizing increasingly spontaneous arranged items could likewise bode well monetarily. 'The huge helpful enhancement and some cost funds might be picked up' by the objective utilization of spontaneously arranged medicines. Pharmacists that did not give compounding administration refered to by the principle reason as not accepting solutions that required compounding $(63.6 \%$ of non-compounder pharmacists). In about $33 \%$ of the aggravated items, they demonstrated patient-explicit reasons. In about $10 \%$ this reason concerned an entirely characterized pharmaceutical consideration issues. A few specialists convincingly said that $73.2 \%$ pharmacists detailed their objective in giving full pharmaceutical consideration to their patients was the most essential helper. The fundamental explanations behind the choice to give compounding administration were need to give full pharmaceutical consideration to patients $(73.8 \%$ of compounder pharmacist) and reacting to demands by prescribers. Most community pharmacists (about 70\%) acknowledged compounding as a part of pharmaceutical consideration. In the greater part of the examinations, real reasons expressed by doctors for endorsing spontaneous items were that they functioned admirably for specific conditions and that the item was not accessible commercially. ${ }^{1-7}$

\section{Evaluation of the Need}

1. Soluble or dispersible tablets may be a useful and convenient alternative to the preparation of liquid extemporaneous products. Some tablets can be dispersed or crushed and information on this aspect can be obtained from the Medicines Information Section within the healthcare setting. In this case, the dose should be prepared and administered immediately. In general, compressed tablets or tablets which are scored or just film coated can be crushed whereas modified release tablets cannot.

2. If a particular medicine is not available as a liquid formulation, another medicine from the same therapeutic classification may well be used, such as the use of a less potent steroid rather than diluting a potent one.

3. Using a suitable preparation intended for a different route of administration, for example, using an injectable solution orally.

4. Use of a 'specials' preparation manufactured in licensed premises (Specials are medicines made in larger volumes by a licensed manufacturer). ${ }^{7}$

\section{Considerations before compounding}

1. Commercially availability of drug in dosage form, strength, and packaging

2. Ingredients, intended use, dosage, and method of administration concern

3. Education, skill and expertise to drug compounding

4. Proper equipment, supplies, chemicals and the guidelines delineated in us pharmacopeia

5. An alternative by which the patient will receive a benefit

6. Safety of the compounded product

7. Patient necessary storage facility, if required,

8. Necessary calculations to prepare the product

9. Necessary documentation to complete preparation

10. Literature reference that might provide information on use, preparation, stability, administration

11. Expected duration of therapy 
12. Some basic quality control to check the product prior to dispensing (e.g., capsule weight variation, $\mathrm{pH}$, visual observations)

13. Ingredient identity, quality, and purity

14. Corrective methods

15. Physico-chemical incompatibilities ${ }^{5,6}$

\section{Economic Considerations}

The most critical factor which limits endorsing of exacerbated medicines was that patients did not need items because of these items were not secured by the national protection. Besides, for specialists not endorsing these items, the significant boundary to recommending was their absence of learning of the items. Most reacting specialists don't endorse medications that require compounding since they need trust in the quality of the intensified details. Notwithstanding, the majority of the specialists communicated a craving to adapt more as appeared in approaching data about the convenience of spontaneous items. There are something like two diverse financial contemplations in settling on the choice to compound medicines; these incorporate (a) pharmacist remuneration and (b) social insurance costs. The evaluating of an exacerbated remedy ought to incorporate thought for pharmacodynamic and pharmacotherapeutic basic leadership, detailing ability, time, and repayment of materials. Compounding solutions can be appealing expertly and monetarily. Compounding solutions can be a method for bringing down the expense of medication treatment. At times, it is more affordable for the pharmacist to set up a particular solution for the patient, which may mean the contrast between the patient really acquiring the medication or managing without it. On the off chance that compounding a remedy results in a patient having the capacity to manage the cost of the medication treatment, it must be considered. An intriguing precedent includes the medication 4aminopyridine. Despite the fact that doctors had been endorsing unapproved variants of the medication for up to 20 years, it was not until the point when the medication was considered efficiently that uncommon seizures were found as a potential reaction. For this situation, the therapeutic calling pushed for an endorsed variant to be promoted, excusing that if a seizure happened with regards to a patient taking a FDA-affirmed elective. ${ }^{4-10}$

\section{Compounding Factors Stability}

One key factor in compounding solutions is strength. The more typical kinds of security of which compounding pharmacists ought to know incorporate concoction, physical, and microbiological. Though commercially made items are required to have a termination date, intensified items are allocated a past use date. There are various wellsprings of data that can be utilized for deciding a suitable past use date, for example, compound organizations, producers writing, research center information, diaries, and distributed books regarding the matter. For the most part, most pharmacists get ready or apportion little amounts of aggravated items; prescribe capacity at room, cool, or cold temperatures; and utilize a traditionalist past use date. For nonaqueous fluids and strong plans (for which the made medication item is the wellspring of dynamic fixing) - The past use date isn't later than $25 \%$ of the time staying until the point when the item's lapse date or a half year, whichever is prior. A USP or NF substance is the wellspring of dynamic fixing-The past use date isn't later than a half year. For water-containing details (arranged from fixings in strong shape) - The past use date isn't later than 14 days when put away at cold temperatures. For every other detailing - The past use date isn't later than the planned span of treatment or 30 days, whichever is prior. These past use date points of confinement might be surpassed when there is supporting legitimate logical solidness data that is specifically appropriate to the particular arrangement (i.e., a similar medication fixation go, pH, excipients, vehicle, water content). For instance, dynamic fixings containing an ester useful gathering, for example, headache medicine and penicillins, are helpless to breakdown by hydrolysis, while those containing aldehyde or hydroxyl gatherings, for example, testosterone and dopamine, experience oxidative decay. $\mathrm{A} 10^{\circ} \mathrm{C}$ increment in temperature can result in a 2-5-overlay quicker rate of corruption. The precariousness of dapsone suspensions arranged in Oral Mix and put away at $25^{\circ} \mathrm{C}$ might be clarified by a Maillard response between the sucrose in the vehicle and the amino gatherings of the dapsone. The aftereffects of this investigation have exhibited the steadiness, for up to 90 days, of dapsone suspensions (2 $\mathrm{mg} / \mathrm{mL}$ ) arranged from business tablets in Oral Mix SF and put away at $5^{\circ} \mathrm{C}$ and $25^{\circ} \mathrm{C}$ or arranged in Oral Mix and put away at $5^{\circ} \mathrm{C}$, in golden plastic jugs and golden plastic syringes. These suspensions ought to be shaken before use. , $, 5,6,11,12^{2}$

Table 1: Common degradation pathways of active drugs in compounded products $* 2,5$

\begin{tabular}{|l|l|l|l|}
\hline Pathway & $\begin{array}{l}\text { Factors } \\
\text { determining } \\
\text { degradation } \\
\text { rate }\end{array}$ & $\begin{array}{c}\text { Susceptible functional } \\
\text { groups }\end{array}$ & \multicolumn{1}{|c|}{ Examples } \\
\hline Oxidation & $\begin{array}{l}\text { Concentration } \\
\text { of drug, } \\
\text { temperature, } \\
\text { catalysts, }\end{array}$ & $\begin{array}{l}\text { Aldehydes, alcohols, } \\
\text { phenols, alkaloids, } \\
\text { unsaturated alkyl chains, } \\
\text { carboxylic acids }\end{array}$ & $\begin{array}{l}\text { Paracetamol, progesterone, testosterone, quinine, oils } \\
\text { (unsaturated fats) such as soybean and corn oil, } \\
\text { essential fats, atorvastatin, atenolol }\end{array}$ \\
\hline
\end{tabular}

IP International Journal of Comprehensive and Advanced Pharmacology, October-December 2018;3(4):124-137 


\begin{tabular}{|l|l|l|l|}
\hline $\begin{array}{l}\text { (O2 } \\
\text { dependent) }\end{array}$ & $\begin{array}{l}\text { solvents, light } \\
\text { and excipients }\end{array}$ & $\begin{array}{l}\text { Esters, amides, lactones, } \\
\text { ethers, lactams, imines, } \\
\text { acetals, anhydrates, } \\
\text { sulfonamides }\end{array}$ & $\begin{array}{l}\text { Aspirin, vigabatrin, norfloxacin, omeprazole, } \\
\text { simvastatin (statins), baclofen, diphenoxylate, } \\
\text { methylphenidate, lignocaine, sildenafil, penicillins, } \\
\text { cephalosporins, diazepam, digoxin, heparin, captopril, } \\
\text { hydrocortisone }\end{array}$ \\
\hline
\end{tabular}

*Drug molecules with more than one functional group can be more easily degraded. In fact, many drugs contain more than one functional group, being susceptible to both oxidation and hydrolysis, e.g. atenolol (contains amide and alcohol groups)

It is essential that the active ingredient does not interact with any excipients originating from the dosage form being crushed for reformulation (e.g. tablet lubricants and fillers), or from additives in the new formulation (antioxidants, preservatives, suspending agents, colour ants, emulsifiers). Interactions with other ingredients can result in physical instability of the product, such as precipitation of the active drug or phase separation ('cracking') of a cream, affecting drug solubility, absorption and bioavailability. To minimize the risk of a compounded medicine degrading, short-term expiry dates are used (e.g. 28 days for oral and topical products, or 24 hours for parenteral formulations), unless stability studies have been conducted and indicate otherwise. ${ }^{2,5}$

\section{Potential Risks}

FDA-endorsed drugs are made and tried as per GMPs, which are government resolutions that oversee the creation and testing of pharmaceutical items. Conversely, exacerbated drugs are absolved from GMPs, and testing to survey item quality is conflicting. Not at all like FDAaffirmed drugs, pharmacy-exacerbated items are not clinically assessed for security or viability. The NABP further portrays compounding as the consequence of a specialist's physician recommended medicate arrange dependent on the expert/tolerant/pharmacist relationship over the span of expert practice. What's more, intensified arrangements don't have standard item naming or recommending data with directions for safe use. Compounding drug stores are not required to report unfavorable occasions to the FDA, which is compulsory for makers of FDA-directed medications. A few drug stores participate in exercises that reach out past the limits of customary pharmacy compounding, for example, extensive scale generation of intensified medications without individual patient solutions, compounding drugs that have not been affirmed for use in the US, and making duplicates of FDA-endorsed drugs. Compounding drugs without GMPs builds the potential for planning mistakes. When compounding is performed on a vast scale, such blunders may antagonistically influence numerous patients. Distributed reports of free testing by the FDA, state offices, and others reliably demonstrate that aggravated medications neglect to meet determinations at an impressively higher rate than FDA-endorsed drugs. Exacerbated sterile arrangements represent the extra danger of microbial defilement to patients. Over the most recent 11 years, three separate meningitis flare-ups have been followed to purportedly 'sterile' steroid infusions tainted with organism or microorganisms, which were made by compounding drug

stores. US-FDA and the NICHD, have cooperated to create item naming for pediatric patients for existing and new medication atoms going to the market, if the medication has been or could be utilized in kids. At the point when contrasted with GMPs, the assembling measures required by FDA for business items, there are various perceived inadequacies characteristic in spontaneous plan compounding appropriate for pediatric patients. ${ }^{11,13}$

Table 2: Why is off-label use of drugs in children still a problem? $?^{13}$

- Lack of specifications required for component development by compounding pharmacies.

- No onsite testing of active ingredients and excipients for purity, potency, content and stability.

- No onsite specifications or testing of product containers and closures.

- Site-to-site variations in compounding procedures, equipment, and the degree of product handling/manipulation.

- Lack of environmental control, which might lead to unintentional contamination and generation of degradation products due to inconsistent exposure to light, temperature and processing controls.

- Lack of testing of finished products for purity, potency, content or stability.

- Stability data for establishing expiry dates of compounded products are derived from published data, where preparation methods likely vary from local methods, or are simply default expiry periods defined by regional pharmacy regulations and "best practices".

- Published preparation methods provide only a portion of the information needed to consistently prepare a stable potent final product.

- Limited options available to mask bad-tasting active ingredients.

- The dose administration technologies used such as droppers, syringes, scoops, spoons, etc., vary between sites and between prescription fills.

- Weak regulatory oversight. 


\section{Quality Control}

One of the quickest developing and most essential regions of pharmaceutical compounding is that of quality control. Quality must be worked in to the arrangement from the earliest starting point ventures to assessing the last planning. The degree of quality control connected to an item ought to be proportionate with the dimension of hazard the completed item could posture to the patient and ought to be made a decision on a case by case premise. Indeed, even where a given definition has been appeared to accomplish reasonable physical, substance and microbiological solidness, the bioavailability and agreeability of the readiness might be doubtful. Thought ought to be given to the individual patient's age and condition, regardless of whether the item will be ingested or utilized topically, just as the strength of, and hazard presented by, the dynamic substances or potentially excipients. Where it isn't handy to complete this testing (for example because of the cluster measure, desperation, and so on.), other reasonable techniques ought to be executed to guarantee that the proper quality is accomplished, as per the hazard appraisal did. At any rate, the beginning materials and completed item ought to be inspected outwardly before supply to a patient. There are a few quality control tests that should be possible inside the pharmacy and others can be sent to an agreement lab. The accompanying quality control tests can be considered for the particular dose shapes.

1. Oral and Topical Liquids (Solutions, Suspensions, Emulsions): Weight/volume, pH, specific gravity, active drug assay, globule size range, rheological properties/pourability, physical observation (color, clarity), physical stability (discoloration, foreign materials, gas formation, mold growth).

2. Hard Gelatin Capsules: Weight-overall average weight, weight individual weight variation, dissolution of capsule shell, disintegration and/or dissolution of capsule contents, active-drug assay, physical appearance (color, uniformity, extent of fill, locked), physical stability (discoloration, changes in appearance).

3. Ointments, Creams and Gels: Theoretical weight compared to actual weight, $\mathrm{pH}$, specific gravity, active drug assay, physical observations (color, clarity, texture-surface, texture-spatula spread, appearance, feel) and rheological properties.

4. Suppositories, Troches, Lollipops and Sticks: Weight, specific gravity, active drug assay, physical observation (color, clarity, texture of surface, appearance, feel), melting test, dissolution test, physical stability.

5. Parenteral Preparations: Weight/volume, physical observation, $\mathrm{pH}$, specific gravity, osmolality, assay, color, clarity, particulate matter, sterility, pyrogenicity. $5,6,14$

\section{Compounding Support}

Numerous agencies, organizations, associations, and so on, are accessible to help pharmacists in compounding.
Data, synthetic concoctions, supplies, and gear are promptly accessible. Substance and supply organizations have expanded in size and number lately and many give data on compounding, contradictions, and stability. Claim to fame compounding associations have created over late years and for the most part give full-line administrations and items to the compounding pharmacist. Numerous national associations give proceeding with expert training programs in both non-sterile and sterile compounding. These substances give administrations to compounding pharmacists going from pitching just compounding helps to giving just synthetic substances. Others offer extra administrations to incorporate formulas just as counseling skill by phone or through the web. This administration can aid the way toward compounding a specific item that might be troublesome.

\section{Table 3: MHRA (2007) in the context of extemporaneous preparation $^{14}$}

- Extemporaneously prepared medicines should be formulated and prepared in compliance with current legal requirements and standards.

- Preparation and quality control arrangements are to be documented and in compliance with current GPP requirements.

- All products prepared should be quality suitable for their intended use

- Products are released for patient use only by a pharmacist.

- Documentation and records should comply recommendations in this guidance document.

\section{Training and Experience}

Pharmacists engaged with redesigning and expanding the conventional parts of impromptu compounding need to keep current with all the new devices of their exchange, recover the old from capacity, and put in a touch of work on utilizing their logical foundation and their craft before they will be agreeable in showing their aptitudes. The pharmacist must guarantee that the staff, offices and frameworks set up are competent on an everyday premise of giving a satisfactory quality administration ready to address the issues of patients. There ought to be a sufficient number of skilled work force consistently. All staff associated with unpremeditated planning must get training Appropriate to their job. Ordinarily, this will: (a) furnish them with learning in great impromptu planning practice, neighborhood works on including wellbeing and security, formulation, expiry periods and quality confirmation proper to the dimension of association, appraisal of hazard and medicine blunder potential, the drug store, its items and administrations gave (b) show competency in the vital unpremeditated readiness aptitudes and pharmaceutical computations and weakenings. Amid training, staff must be cautiously administered and checked and students must comprehend the breaking points of their obligations. While considering giving extra administrations of compounding in a foundation, pharmacists ought not expect that this will 
change a lot of their training in time devoured for compounding. A composed training project ought to be accessible and finish of training ought to be recorded. This applies to all staff working in the readiness region, including those not specifically associated with arrangement forms (for example cleaning staff). ${ }^{14}$

\section{Equipment}

The equipment required will be dictated by the sort and degree of the administrations one gives. Numerous drug stores as of now have clean air conditions (e.g., laminar wind stream hoods, detachment boundary frameworks) where aseptic compounding of sterile arrangements is performed. These equivalent units can be utilized to compound other sterile arrangements, for example, eye drops. An equalization, ideally electronic, is fundamental. Balm sections (i.e., pill tiles), alongside spatulas of various kinds and materials, ought to be bought. A couple of mortars and pestles (i.e., glass, fired, plastic) and some dishes ought to be anchored. An arrangement for the isolation of explicit gear for certain item types, including cytotoxic medications and outer items, ought to be accessible; this should consider nearby COSHH appraisals. Hardware ought to be adjusted and approved to show attractive execution. Measures ought to be adjusted for explicit volumes and ought to have leveling lines. Dish sets ought to be reviewed consistently, when each utilization, for chips and splits, and supplanted as essential. Thought ought to be given to the benefits of different materials. Hardened steel of a reasonable review is a decent material yet care must be taken with a few specialists as it is vulnerable to oxidation. Plastics are helpless to surface scratches and stains. The exactness of parities ought to be kept an eye all the time and records of this procedure kept up. It may not be important to purchase a roomful of hardware; however, one should buy what is expected to begin the administration and should expand on it as the administration develops and extends to various fields. A great part of the gear utilized today in compounding has changed. Today, electronic adjusts are utilized more regularly than torsion adjusts; miniaturized scale pipets are ordinary; and ultra-coolers are here and there required notwithstanding standard icebox coolers. This region is always showing signs of change and the compounding pharmacist ought to know about the accessible innovation to get ready exact and powerful solutions. Getting to be familiar with the nearby delegate for a research center supply organization is useful.,2,6,14-19

\section{Environment}

A different region for conventional compounding is prescribed, as opposed to just wiping off a little zone of the apportioning counter. The compounding pharmacist needs a spotless, flawless, sufficiently bright and calm working zone. On the off chance that aseptic compounding is considered, a spotless air condition (e.g., laminar wind stream hood, segregation hindrance framework) ought to be utilized. The real office to be utilized relies upon the dimension and volume of compounding to be finished.
Impromptu compounding happens in network and healing facility drug stores. There are normally pro compounding drug stores in significant towns and urban areas, yet any drug store may attempt compounding as long as they have fitting offices as per state-based enactment (for example distributed clean seat, explicit compounding hardware). There ought to be a committed office/space for unpremeditated planning. Be that as it may, for drug stores planning little amounts of a constrained scope of items for quick administering then an assigned zone or incidentally assigned zone is admissible. The territory must be plainly outlined amid planning. Measures must be gone out on a limb of cross-defilement. Just a single item ought to be taken care of at once. Premises and hardware ought to be anything but difficult to clean. Following support or fix, intensive cleaning and, where fitting, sterilization should occur. Ecological conditions, including temperature, moistness and lighting, must be satisfactory to allow protected and agreeable arrangement of restorative items to constrain the corruption of fixings. Offices ought to be spread out in an approach to give sufficient space to the exercises embraced. Work should stream legitimately between working zones without the danger of item crosspollution. Complex compounding is performed in a pressurized clean room utilizing a laminar stream bureau, cytotoxic medication security bureau or an isolator. Numerous open and private clinics keep up extensive aseptic compounding offices to give individualized dosing or industrially inaccessible formulations. Territories and hardware ought to be cleaned adequately with an appropriate cleanser when use. Gear ought to be flushed with a suitable review of water in the wake of washing (consumable or sterile water for water system/infusion) and afterward dried. Basic surfaces ought to be successfully purified with an appropriate specialist, for example, 70\% liquor before use. Sinks ought to be accessible for cleaning up of gear, with discrete offices for handwashing. Sinks ought to be a reasonable separation far from the planning zones to decrease the danger of microbial pollution from water sprinkles. Washing and cleaning exercises ought not themselves be a wellspring of tainting. All gear ought to be obviously perfect preceding use. Guarantee that all buildups of cleaning specialist have been expelled Containers and tops ought to be checked to guarantee that they are spotless and dry before use. Sufficient irritation control measures ought to be taken. ${ }^{6,10,19}$

\section{Formulas}

Consistency of the compounded product is important. Formulas should be developed or obtained and tried to assure that each time an extemporaneous product is prepared, the methods used, ingredients added, and the order of steps is documented. This accomplishes three things. First, it provides the methodology for each person involved or requested to provide such service the information necessary to do so properly. Second, it provides consistency from batch to batch. Third, if the product does not turn out the way expected, a stepwise methodology exists for 
reviewing and determining what happened and if revisions and improvements are needed. ${ }^{6,15}$

\section{Chemicals and Supplies}

If one is going to prepare a topical product, a vehicle (e.g., cream, ointment, gel) and the active ingredients (e.g., either finely ground product from an available tablet or injection or pharmaceutical-grade chemicals) would be required. One needs proper dispensing containers for the medication. In short, a relationship with providers that carry chemicals and supplies is important. Pharmacists have been using chemicals and other materials for prescription compounding throughout history. In the past, these chemicals and materials have been obtained from natural products, raw materials, and household ingredients. Today, compounding pharmacists use chemicals from various reliable commercial sources, depending on their availability. ${ }^{9,15-18}$

\section{Documentation}

These give preparing, bundling and discharge guidelines. For items routinely arranged, ace archives which join the detail and item explicit guidelines ought to be created and autonomously checked. Then again, a basic last item particular might be delivered notwithstanding the ace archive. Ace records ought to be clear and point by point and ought to include an institutionalized style inside any one drug store. Basic stepwise directions for arrangement, alongside a particular notes or alerts (for example $\mathrm{COSHH}$ prerequisites) ought to be incorporated on the ace record for every item. A record ought to be kept of the key phases of handling, bundling and arrival of items to give a review trail of the quality applicable realities of the historical backdrop of a spontaneous item amid planning. This is typically accomplished by fruition of an individual worksheet, as a rule by photocopying the ace archive. Worksheets will shift for every drug store yet ought to include: the name and formula of the item, and the wellspring of the formula; an exceptional ID number to empower recognizability; the maker, cluster quantities of each beginning material (or QC reference number); the date of readiness and so forth. Names must conform to all statutory and expert prerequisites, and ought to incorporate the accompanying data: the name of the item, and where vital the idea of the salt and any waters of crystallization; amount and quality of dynamic fixings; the pharmaceutical shape and so forth. ${ }^{6,20}$

\section{Types of Compounding}

Active pharmaceutical ingredients can be incorporated into a wide array of products including creams, eye drops, nasal sprays, oral dosage forms or intravenous infusions. In Australia, products may be classified into simple or complex compounding (Table 1). Simple compounding can be performed by any pharmacist and is a core competency of pharmacy training. Complex compounding requires additional training and evidence, as described by the Pharmacy Board of Australia's guidelines on compounding. Finland, Italy, and Scotland prepared mainly powder, while Belgium, Croatia, France, and Switzerland prepared mainly capsules. Practice in Germany, Spain, and Slovenia involved preparation of a less welldefined combination of liquid, powders, and capsules.

Table 4: Classification of simple versus complex compounding ${ }^{5}$

\begin{tabular}{|c|c|c|}
\hline Type & Explanation & Examples \\
\hline Simple & $\begin{array}{l}\text { All pharmacists have training during their } \\
\text { undergraduate degree to prepare these } \\
\text { products. } \\
\text { Involves well-established preparations } \\
\text { published in reputable literature, e.g. the } \\
\text { Australian Pharmaceutical Formulary and } \\
\text { Handbook, or formulae for which some data } \\
\text { are available regarding quality, stability, } \\
\text { safety, efficacy and rational design. }\end{array}$ & $\begin{array}{l}\text { Topical creams, ointments, lotions, gels, e.g. steroids, } \\
\text { hormones, coal tar, cholestyramine. } \\
\text { Oral liquids (solutions, suspensions, emulsions, mixtures, } \\
\text { elixirs), tinctures, e.g. omeprazole suspension. Capsules, } \\
\text { tablets, powders, e.g. ethinylestradiol capsules. } \\
\text { Suppositories, pessaries, e.g. paracetamol, clotrimazole }\end{array}$ \\
\hline Complex & $\begin{array}{l}\text { Pharmacists require further postgraduate } \\
\text { training in association with self-assessment } \\
\text { of relevant competencies and documentation } \\
\text { of the specific competencies in a continuing } \\
\text { professional development plan. Specialized } \\
\text { facilities (sterile room with positive } \\
\text { pressure) and equipment (laminar flow } \\
\text { isolator, dry heat sterilization oven) are also } \\
\text { required. }\end{array}$ & $\begin{array}{l}\text { Parenterals, e.g. morphine, clonidine. Ingredients with a } \\
\text { safety hazard, e.g. cytotoxics, hormones } \\
\text { Single unit micro-dose }(<25 \mathrm{mg} \text { of drug or no more than } \\
25 \% \mathrm{w} / \mathrm{v} \text { of a dosage form), e.g. naltrexone. Modified- } \\
\text { release dosage forms, e.g. levothyroxine (T4), } \\
\begin{array}{l}\text { progesterone } \\
\begin{array}{l}\text { Ophthalmic preparations, e.g. phenylephrine, } \\
\text { tropicamide, ciprofloxacin }\end{array}\end{array}\end{array}$ \\
\hline
\end{tabular}

\section{Ambulatory-Care Compounding}

In the event that people can walk, they are viewed as versatile or wandering (i.e., they are not out of commission). Thusly, most pharmacists are associated with wandering consideration, and most mobile patients are outpatients. As a matter of fact, the term can likewise be connected to home-care patients and even organized patients who are versatile. One general normal for wandering patients is that they are commonly in charge of acquiring their very own prescription, putting away it, setting it up (if essential), and 
taking it. It appears to be practically muddled that in medicinal services today as we turn out to be increasingly mindful that patients are people, react as people, and should be treated as people that some social insurance suppliers give off an impression of being gathering patients into classes. They are gathered in classes for treatment, for repayment from an outsider, or for deciding dimensions of consideration in oversaw care associations and utilizing settled portion items given by pharmaceutical makers that are accessible in light of the fact that the promoting request is adequately high to legitimize their make and creation. For what reason should the accessibility or the absence of accessibility of a particular financially beneficial economically accessible item manage the treatment of a patient? Pharmacists have a chance to expand their exercises in patient consideration as the accentuation keeps on moving from inpatient care to mobile consideration. Wandering consideration, in any case, is so assorted and includes such a large number of orders that occasionally it is hard to comprehend it; and, it changes quickly. Additionally, wandering consideration could for the most part urge a group way to deal with wellbeing enhancement, anticipation, wellbeing upkeep, hazard evaluation, early location, the executives, healing treatment, and restoration. Wandering consideration offers different open doors for individualizing tolerant consideration through pharmaceutical compounding. In fact, it is the area where most compounding pharmacists' practice. Pharmacists' roles in ambulatory care patients can include, among others.

1. Dispensing

2. Compounding

3. Counseling

4. Minimizing medication errors

5. Compliance enhancement

6. Therapeutic drug monitoring

7. Minimizing expenditures

Most reimbursement for ambulatory patients comes from the dispensing or the compounding process. Little financial consideration is given to counseling, minimizing medication errors, compliance enhancement and therapeutic monitoring. However, these activities are important and should be performed. Because of the unique nature of compounded medications, counseling is an absolute must for these patients. From the above discussion of the activities of ambulatory care pharmacists, it should be evident that extemporaneous compounding can be vitally important in ambulatory patient care. ${ }^{6,18-21}$

\section{Hospital Pharmacy Compounding}

The ever-present obligation of the social insurance industry is to give the best accessible consideration to the patient, utilizing the best way to do as such, and giving that care in a helpful domain. This must be adequately efficient to not put the foundation in risk of being not able keep on giving the administrations to the network they serve. This requires participation with respect to the clinic organization, the medical staff, and the representatives (attendants and pharmacists specifically as respects to prescription utilization) and must include the patient. One of the viable methods by which clinics, and in this manner healing facility drug stores, can address these difficulties is to consider extending unpremeditated compounding administrations inside the doctor's facility drug store. Pharmaceutical consideration and pharmaceutical compounding can give cost investment funds to the clinic while giving required choices to the doctor through critical thinking approaches and animating the healing center pharmacist through new difficulties that permit the statement of both their abilities and their specialty. ${ }^{15,22,23}$

\section{Veterinary Compounding}

The first symposium on veterinary compounding was a significant forum for discussion by experts and was a pivotal point in the history of veterinary compounding, occurring in September 1993. the meeting was important because it assembled an impressive group of experts on veterinary compounding, who then set about explaining and defining the roles of the veterinarian and the pharmacist. ${ }^{24}$

\section{Nuclear Pharmacy Compounding}

Nuclear pharmacy is a specialty practice of pharmacy that has been defined as a patient-oriented service that embodies the scientific knowledge and professional judgment required for improving and promoting health through assurance of the safe and efficacious use of radioactive drugs for diagnosis and therapy. Radioactive drugs, commonly referred to as radiopharmaceuticals, are a special class of drugs that are regulated by the FDA. They are unique in that they contain an unstable nuclide (radioactive nuclide) as a part of the compound designed to localize in an organ or tissue. Since radiopharmaceuticals are radioactive, the Nuclear Regulatory Commission or a similar state agency is involved in regulatory matters relevant to radiopharmaceuticals..$^{25-27}$

\section{Risk Management}

Legal Background: Pharmacists must comply with all legislation relevant to the practice of pharmacy in the jurisdiction where the practice occurs. Failure to practice in accordance with these requirements may give rise to action by one or more responsible authorities. These matters may then be referred to the Board for appropriate action under the National Law or law of a co-regulatory jurisdiction. Non-compliance with these guidelines and the practice standards and guidelines relevant to compounding may also be notified directly to the Board for appropriate action under the National Law or law of a co-regulatory jurisdiction. Non-compliance may be reported by an individual lodging a notification form, or through other means such as notification of outcomes of audits carried out by a state/territory pharmacy premises regulatory authority or responsible body. ${ }^{5}$ 
Table 5: Examples of pre-1938 drugs that remained on the market as unapproved drugs ${ }^{15}$
Acetaminophen, codeine phosphate, and caffeine capsules and tablets
Amobarbital sodium capsules
Amyl nitrate inhalant
Chloral hydrate capsules, syrup, and suppositories
Codeine phosphate injection, oral solution, and
tablets
Codeine sulfate tablets
Colchicine injection and tablets
Digitoxin tablets
Digoxin elixir and tablets
Ephedrine sulfate capsules and injection
Ergonovine maleate injection and tablets
Ergotamine tartrate tablets
Hydrocodone bitartrate tablets
Hydrocodone bitartrate, aspirin, and caffeine tablets
Hydromorphone hydrochloride suppositories
Levothyroxine sodium for injection

\section{Alternatives to Extemporaneous Preparation}

1. Therapeutic substitution: The utilization of an authorized prescription from a similar restorative order ought to be considered and may give a superior clinical choice than the utilization of a without premeditation arranged medication which has restricted information to help its formulation and stability. The utilization of a less intense steroid instead of weakening a strong operator is a model where a helpful option may dispose of the requirement for an unpremeditated arrangement. There are incredible open doors for oncology pharmacists, just as network pharmacists, as an asset for instructing and observing patients accepting oral chemotherapy to guarantee dosing exactness, safe organization, and appropriate transfer of dangerous medications. Oncology pharmacists confront a consistent test with patients who can't swallow oral anticancer medications, making unpremeditated oral fluid readiness a necessity. Ill-advised spontaneous arrangement of these specialists, particularly with the conventional chemotherapy with a limited restorative record, may expand the danger of over-or underdosing. ${ }^{28}$

2. Use of an Imported Product: The preparation selected should be licensed for use in a country with equivalent or similar licensing arrangements and regulatory standards to the UK (e.g. EU, Canada, Australia). This will provide the requesting pharmacist with assurance that the quality, safety and efficacy of the medicine have been reviewed by a competent regulatory authority. Although clinicians do not doubt the efficacy and utility of captopril in managing children with heart failure, concerns exist about optimal dosing schedules and toxicity. This formulation is licensed in the Bristol-

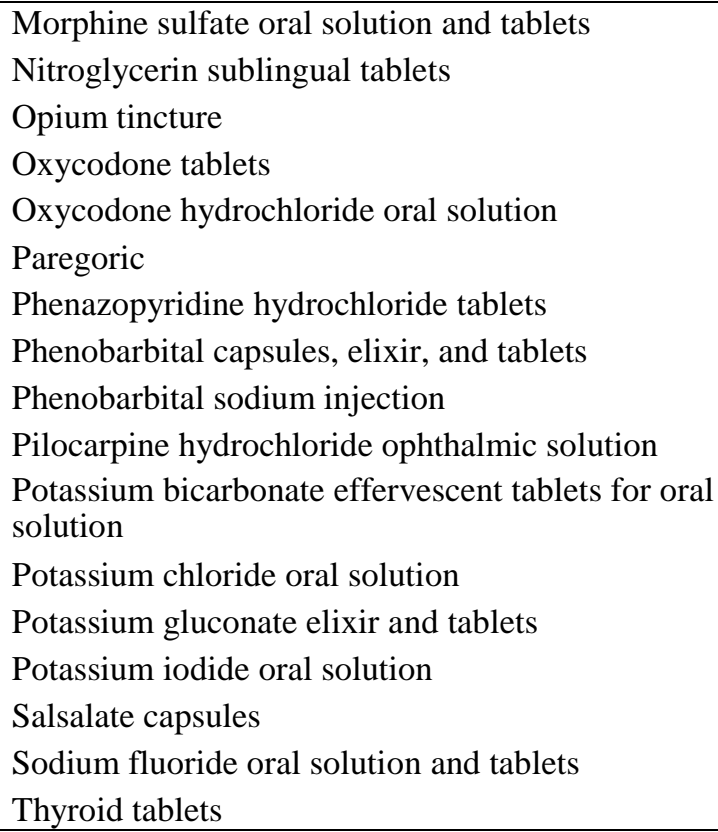

Myers Squibb, Australia, but was not available in the $\mathrm{UK}^{29}$

3. Use of a 'Special' Manufactured in a MHRA Licensed Unit: The benefit of purchasing a 'Special' is that the product should be made to a validated formula with supporting stability data in accordance with the principles of GMP. Licensed 'Specials' units are regularly inspected by the MHRA to ensure these principles are upheld. However, the purchasing pharmacist will still need to review the supporting documentation (e.g. specification, Certificate of Analysis/Conformity, BSE/TSE statement) to assess whether the product is of appropriate quality. Information on Specials manufacturers is available in the BNF. Specials may be used to meet a variety of patient needs, for example, for babies, children and older people, and in specific areas such as dermatology and palliative care. ${ }^{30}$

4. Use of Soluble or Dispersible Tablets: This practice presents fewer health and safety risks than crushing tablets, which can expose the carer to potentially harmful dusts via inhalation. When dispersing tablets, the dose should be prepared and administered immediately, as stability cannot be guaranteed. It should be noted that slow or modified release preparations should not be used in this manner. If the active is not soluble, it can lead to inaccuracy of dosing through a lack of dose uniformity and reproducibility. This is a major consideration when no suspending agents are used, especially when the person administering the dose is inexperienced and the dose is small, ${ }^{31}$ Milk has been explored as a vehicle in liquid formulations showing potential for solubilizing drugs while maintaining the stability of the emulsified vehicle. $^{32}$ 
5. Cutting Tablets: Tablets cannot be cut with great accuracy of dose and research suggests that the variability may range from $50 \%$ to $150 \%$ of the desired dose even when using commercially available tablet cutters.

6. Use of a Preparation Intended for a Different Route: The use of a suitable preparation intended for a different route of administration can sometimes be a practical alternative; for example, the use of an injection solution orally, or an oral solution rectally. However, this practice has its own inherent risks and the pharmacist should ensure that the presentation used will be absorbed by this route and that it will be tolerated by the patient. When using an injection by the oral route, consideration should be given to the possibility of rapid absorption and elevated peak levels, the potential for rapid drug degradation due to exposure to gastric acid and problems with first-pass metabolism. The $\mathrm{pH}$ of an injection should also be considered, as extremes of $\mathrm{pH}$ can adversely affect the gastric mucosa. ${ }^{20}$

\section{Risks associated with Extemporaneous Preparation}

1. Formulation Failure: An insoluble drug suspended in a suitable vehicle may be less susceptible to drug degradation, but may settle out of the suspension over time, leading to sedimentation and caking. In this state, there will be a higher concentration of drug at the bottom of the bottle than at the top. In 2006, the FDA conducted a limited survey of compounded drugs. Of 36 samples tested by the FDA, 12 failed at least one quality test, for a failure rate of $33 \%$. Further, oral hormone dosage forms containing multiple active ingredients showed poor content uniformity, with random variation in all three active ingredients from capsule to capsule. ${ }^{8,20}$

2. Microbial Contamination: The decision of additive for a formulation needs to consider various variables including $\mathrm{pH}$, physical similarity and the expected patient gathering. Unpreserved arrangements ought to be put away in an icebox and doled out a short time span of usability to confine microbial development. A greatest timeframe of realistic usability of 7 days at 2 $80 \mathrm{C}$ ought to be doled out to unpreserved oral fluid arrangements except if adequate approval work has been done to help an all-inclusive time span of usability. Basic macronutrients and micronutrients imbued by means of PN arrangements are a fundamental piece of the consideration of babies and kids who can't or ought not be nourished enterally. Babies and kids require a more noteworthy measure of fat in their eating regimen than do grown-ups to help development and improvement. The utilization of IVFE gives a concentrated wellspring of calories and keeps the advancement of basic unsaturated fat lack in neonates encouraged only by means of the parenteral course. The AAP prescribes IVFE dosages of up to 3 $\mathrm{g} / \mathrm{kg} /$ day (around $30 \mathrm{kcal} / \mathrm{kg} /$ day) for use in babies. An assortment of microorganisms has been found in lingering IVFE after culmination of the mixture. ${ }^{33}$ Microorganisms could conceivably be presented amid reformulation of non-sterile items. For instance, if Candida albicans is unintentionally brought into newly arranged multi-portion citrus extract arrangements that are to be utilized orally for hack reflex testing, immunocompromised people could wind up contaminated. Normal checking of the earth, hardware and techniques is fundamental to guarantee quality and sterility is kept up. The outcomes of disappointment can be disastrous, for instance methylprednisolone infusions from a solitary compounding drug store in the USA brought about 137 instances of Aspergillus fumigatus meningitis and 12 death. ${ }^{2}$

3. Calculation Errors: Normal computation blunders related with unpremeditated arrangement incorporate mistakes while changing over units starting with one then onto the next (for example milligrams to micrograms, changes from load in volume to millimoles). Risks of strong fixings and computation blunders, especially where the quality of at least one fixing is expressed in a recorded or non-standard mold. Comparable reports have started from the US, with the demise of a tyke from a super-powerful imipramine fluid, and a five-year-old tyke who got a 1,000-overlap overdose of clonidine. Issues can likewise emerge when portions can be recommended as free base or salt, prompting potential count blunders when making and regulating arrangements (for example two-overlay blunders if caffeine citrate is mistaken for caffeine base). A portion of these blunders have been ascribed to conflicting marking approaches, for instance, qualities communicated per milliliter or per $5 \mathrm{ml}$ spoonful. Clinical pharmacists in pediatrics and neonatology help in portion computation and measurement frame alteration. The expanded requirement for computations and weakening of pediatric meds, and a prerequisite to alter portion of an individual patient dependent on age, gestational age, weight and surface zone, give a more prominent chance to a clinical pharmacist in medicine the executives procedure. They can get ready pediatric/neonatal formulary. ${ }^{34,41}$

4. Starting Materials: Care should also be taken with the use of cariogenic sugars (e.g. sucrose) in pediatric formulations as it has been associated with dental cavities. It is therefore important to list all such excipients on the product label so that end-users are made aware of their presence in the formulation. In a 2004 published analysis sponsored by STD Pharmaceuticals, all samples purchased from three compounding pharmacies failed content testing for a $3 \%$ sodium tetradecyl sulfate solution for injection (range 2.59-3.39). Significant concentrations of the contaminant carbitol were found to be present in samples from all three sources (0.33-4.18), suggesting possible use of a non-pharmaceutical grade chemical..$^{35}$ 
5. Patient Acceptability Issues: Thought ought to be given to the agreeability and introduction of oral fluid medications as there is a decent contention that taste is pivotal to accomplishing great consistence in youngsters, particularly for the treatment of longstanding conditions, for example, in cardiology. On the spur of the moment arranged oral medication formulations can likewise be tormented by poor acceptability qualities which can trade off patient adherence. This absence of kid well disposed formulations influences $40 \%$ of the worldwide populace, exposing pediatric patients to avoidable unfavorable medication occasions, diminished consistence with medicine regimens, restricted access to new drugs, and delayed treatable ailments. The squeezing requirement for kid benevolent, acceptable drugs appropriate for organization to the two newborn children and youthful youngsters has been worried (through administrative direction) by both the European Medicines Agency and the US FDA. Prednisone is an unpleasant tasting corticosteroid utilized for its mitigating and immunosuppressant impacts to treat a wide assortment of conditions in the two grown-ups and youngsters. For pediatric patients, an elective type of organization can be delivered by pounding strong oral prednisone formulations so as to abstain from gulping troubles as well as accomplish weight-based dosing. This methodology, notwithstanding, makes amazingly poor taste and mouth feel properties which are questionable to pediatric patients. While these impediments can be to some degree alleviated by the utilization of exclusive oral fluid formulations of prednisone, such items still hold their significantly harsh taste attributes. It is assessed that around half of kids decline to take even a fluid type of prednisone, with the vast greater part of those revealing terrible taste as the single real purpose behind resistance. Endeavors to cover flavors utilizing improving specialists, coatings, agglomeration, or microencapsulation frequently result in inadequately controlled, heterogeneous molecule estimate dispersions that outcome in an abrasive or granular mouth feel and can give ineffectual taste concealing, attributes that may likewise bargain persistent acknowledgment. The structure of a perfect pediatric formulation needs to think about the accompanying elements: (I) creating negligible effect on the way of life of the tyke, showing as the most reduced measurements recurrence and an agreeable item, (ii) arrangement of individualized dosing or portion banding fitting for powerful treatment, (iii) adequate bioavailability, (iv) non-dangerous excipients in the formulation, (v) helpful and solid organization and (vi) strong generation process at insignificant expense. ${ }^{20,36-39}$

6. Health and safety Risks: When handling hazardous products, units should be equipped with suitable containment devices and systems should be put in place to eliminate the risk of cross-contamination.
7. Therapeutic Risks and Clinical Consequences: Any inaccuracy of dosing associated with medicines that have a narrow therapeutic index can lead to significant morbidity, whether due to under-dosing leading to treatment failure or overdosing leading to toxicity. By contrast, any inaccuracy of dosing associated with drugs with a wide therapeutic index may have little or no impact on the therapy.

8. Associated Clinical Risk Factors: The majority of patients receiving extemporaneously prepared products, in particular oral liquid medicines, tend to be from vulnerable patient groups (e.g. neonates, children, stroke victims) who are either unaware of ill-effects associated with their treatment or who cannot communicate with their clinician. Coupled with this, extemporaneous preparations may not be routinely identified as high-risk therapies by pharmacists and therefore such treatments are not commonly given the level of scrutiny and close monitoring they require. Therefore, when embarking on the use of an extemporaneously prepared product, the pharmacist should ensure that systems are in place to monitor the effectiveness of the therapy. ${ }^{20}$

\section{Risk Management}

The European Pharmacopeia monograph on Pharmaceutical Preparations indicates the need for tests to be connected to specific dose shapes, as portrayed in their particular measurement frame monographs, which are additionally appropriate on account of on the spur of the moment arranged items. The prerequisites on testing in the European Pharmacopeia monograph manage the presence of items, character and immaculateness tests, consistency and reference measures. The degree of value control connected to an item ought to be proportionate with the dimension of hazard the completed item could posture to the patient and ought to be made a decision on a case by case premise. Thought ought to be given to the individual patient's age and condition, regardless of whether the item will be ingested or utilized topically, just as the power of, and chance presented by, the dynamic substances as well as excipients. Where it isn't functional to complete this testing (for example because of the cluster measure, criticalness, and so on.), other reasonable strategies ought to be actualized to guarantee that the fitting quality is accomplished, as per the hazard evaluation did. At the very least, the beginning materials and completed item ought to be inspected outwardly before supply to a patient. ${ }^{40}$

\section{Role of the Pharmacists}

Compounding arrangements without premeditation on legal remedy for individual patients is inside the pharmacist's domain. Pharmacists and drug stores are authorized and examined by the states; if a state investigation reveals "producing under the pretense of compounding," it alludes the issue to the FDA, which can apply cGMP desires to the pharmacist/drug store. At the point when a pharmacist participates in vast scale creation 
of arrangements without medicine or proof of pending refills, drifting, and so forth, the training can be viewed as assembling; cGMP consistence will be normal, including an endorsed NDA or ANDA. Today, be that as it may, compounding drug stores have started to thrive and are by and by turning into an indispensable part of drug store practice. Numerous social insurance experts perceive that, for some particular patient populaces, there is a developing need to exacerbate certain drugs since they are not accessible through ordinary assembling strategies. Though the administrations given by compounding drug stores give numerous patients alternatives with respect to sedate treatment, compounding pharmacists must be legitimately prepared to represent considerable authority in this part of drug store practice and should have the proper assets and references to compound quality prescriptions. Pharmacists are in charge of guaranteeing that spontaneous arrangements are aggravated by compounding rules and norms concerning immaculateness, quality, stability, pressing, record keeping, and other fitting drug store rehearses. Compounding pharmacists must work intimately with the endorsing doctor to guarantee that understanding objectives are met. A positive remedial result for the patient is the basic objective though wellbeing couldn't care less experts, and, in light of that objective, the administrations given by compounding drug stores can keep on making pharmacists a key piece of the medicinal services group. ${ }^{42,43}$

\section{Conclusion}

Pharmacy compounding provides pharmacists with a unique opportunity to practice their time-honored profession. It is becoming an even more important part of pharmacy practice in the future, including those involved in community and hospital care, nursing care, home care, veterinary and other specialty practices. It is a practice where the clinical expertise can be merged with the scientific expertise of pharmacists to make a visible pharmaceutical care. Pharmacists are to be encouraged more in compounding but should be aware of the core of formulating a specific drug product for a specific patient. This is important in providing pharmaceutical care.

\section{Conflict of Interest: None.}

Abbreviations: Good Compounding Practices (GCPs); Joint Commission on Accreditation of Health Care Organizations (JCAHO); National Association of Boards of Pharmacy (NABP); Control of Substances Hazardous to Health (COSHH); Extemporaneous preparations (EPs); National Institute of Child Health and Human Development (NICHD); Medicines and Healthcare products Regulatory Agency (MHRA); Transmissible Spongiform Encephalopathy (TSE); Bovine Spongiform Encephalopathy (BSE); Intravenous Fat Emulsion (IVFE); American Academy of Pediatrics (AAP); National Association of Boards of Pharmacy (NABP); New Drug Application (NDA); Abbreviated New Drug Application (ANDA).

\section{Article Highlights:}

1. An estimate by FDA that $1-10 \%$ of all prescriptions require compounding was not supported by evidence data.

2. In 2006, the FDA conducted a limited survey of compounded drugs revealed failure rate of $33 \%$.

3. In 2001, a Kansas City-based pharmacist was discovered to have adulterated 72 different drugs, including many oncology medications, to increase profits.

4. Over the period 2008-2010, the Texas State Board of Pharmacy found an overall potency failure rate of $23 \%$ for compounded drugs

5. Methylprednisolone injections from a single compounding pharmacy in the USA resulted in 137 cases of Aspergillus fumigatus meningitis and 12 deaths.

6. Even where a given formulation has been shown to achieve suitable physical, chemical and microbiological stability, the bioavailability and palatability of the preparation may be unproven.

7. Ingredients, intended use, dosage, and method of administration, education, skill and expertise; proper equipment, supplies, chemicals the guidelines delineated in US pharmacopeia, necessary calculations and documentation; expected duration of therapy, physico-chemical compatibility are the major considerations before compounding.

8. Quality must be built-in to the preparation from the beginning steps to evaluating the final preparation.

9. The use of an alternative route of administration (use of the rectal rather than the oral route) could also be considered if an appropriate formulation is available.

\section{Article Synopsis}

It is important to use a pharmacist's expertise to adjust dosage quantities, frequencies, and even dosage forms for enhanced compliance. All pharmacists should understand the options presented by compounding. Pharmaceutical compounding is increasing for a number of reasons, including the availability of a limited number of dosage forms for most drugs, a limited number of strengths of most drugs, home health care, hospice, the non-availability of drug products/combinations, discontinued drugs, drug shortages, orphan drugs, new therapeutic approaches and special patient populations. Extemporaneously compounded medicines may be useful when a required dose or dose form is unavailable commercially, or for individualized dosing. There are numerous established compounding formulae available, and new formulae may be developed with the help of formulation guidelines and professional advice. Unlike registered medicines, compounded preparations have not generally been assessed for safety and efficacy. Their use is off label and is based on extrapolation from the component ingredients. Short-term expiry dates are provided for compounded products unless their stability has been assessed. 


\section{References}

1. Taylor S, Hays C, Glass B. Compounding medications in a rural setting: an interprofessional perspective. J Multidiscip Healthc 2018;11:197-204. Published 2018 Apr 20. doi:10.2147/JMDH.S156161

2. Falconer JR, Steadman KJ. Extemporaneously compounded medicines. Aust Prescr 2017;40(1):5-8.

3. Gullapalli RP, Mazzitelli CL, Charriez CM, Carpenter DJ, Crean RD, Carter B, Perera P. Extemporaneous preparation strategy for early phase clinical studies. Int J Pharm 2018;549(1-2):150-160. doi: 10.1016/j.ijpharm.2018.07.059. Epub 2018 Jul 25. PubMed PMID: 30055303.

4. Brown G. The Value of Drug Stability Studies and Their Publication. Can J Hosp Pharm 2018;71(3):161-162.

5. Pharmacy Board of Australia. Codes, guidelines and policies. Guidelines on compounding medicines. 2015. http://www.pharmacyboard.gov.au/Codes-Guidelines.aspx

6. Allen VL. Chapter 105. Extemporaneous Prescription Compounding. In: Remington: The Science and Practice of Pharmacy by David B. Troy, Paul Beringer, published by Lippincott Williams \& Wilkins, 2006

7. Costello, Ian; Long, Paul; Wong, Ian Chi Kei; Tuleu, Catherine; Yeung, Vincent. Paediatric Drug Handling. London: Pharmaceutical Press; 2007

8. Sellers S, Utian WH. Pharmacy compounding primer for physicians: prescriber beware. Drugs 2012;72(16):2043-2050.

9. Loyd VA. The Art, Science and Technology of Pharmaceutical Compounding Fifth Edition APhA Wshington, D.C.

10. Ivana Jaksic, Milica Lukic, Andjelija Malenovic, Stephan Reichl, Christine Hoffmann, Christel Müller-Goymann, Rolf Daniels and Snezana Savic, Compounding of a topical drug with prospective natural surfactant-stabilized pharmaceutical bases: Physicochemical and in vitro/in vivo characterization A ketoprofen case study, European Journal of Pharmaceutics and Biopharmaceutics, 80, 1, (164), (2012).

11. Gudeman J, Jozwiakowski M, Chollet J, Randell M. Potential risks of pharmacy compounding. Drugs $R$ D 2013;13(1):1-8.

12. Alarie H, Friciu MM, Leclair G. Stability of Dapsone in Extemporaneously Compounded Oral Suspensions. Can J Hosp Pharm 2018;71(2):152-154.

13. Bhatt-Mehta V, MacArthur RB, Löbenberg R, Cies JJ, Cernak I, Ii RHP. An Algorithm to Identify Compounded Non-Sterile Products that Can Be Formulated on a Commercial Scale or Imported to Promote Safer Medication Use in Children. Pharmacy (Basel). 2015;3(4):284-294. Published 2015 Nov 11. doi:10.3390/pharmacy3040284

14. Handbook of Extemporaneous Preparation: A Guide to Pharmaceutical Compounding Rowe, Lesley (Reviewed by) Edited by Mark Jackson, Published by the Pharmaceutical Press, London, Chicago. Aust Pharm 2010;29(10).

15. Allen VL. Chapter 1. Guidelines for Compounding Practices. In: Loyd V. Allen. The Art, Science, and Technology of Pharmaceutical Compounding, 5th Edition, publisher American Pharmacists Association 2017

16. Extemporaneous formulations reference guide. The Pharmaceutical Journal 24 JUN 2016 By Laurence A. Goldberg

17. Medicines, Ethics, and Practice: A Guide for Pharmacists, Volume 29 by Royal Pharmaceutical Society of Great Britain, 2005

18. McHugh MB. Chapter 15. Non-sterile compounding and Repackaging. In: Mary B. McHugh. Workbook for the Manual for Pharmacy Technicians, published by ASHP, Jan 1, 2013

19. USP DI., Volume 1, Parts 1-2; Volumes 2-3 by United States Pharmacopeial Convention, 2006

20. Handbook of Extemporaneous Preparation: A Guide to Pharmaceutical Compounding by Mark Jackson, Andrew Lowey, published by Pharmaceutical Press, 2010
21. The Pharmacy Technician by Jahangir Moini, published by Cengage Learning, Mar 3, 2010

22. Sundberg JA. Extemporaneous Compounding in the Hospital Pharmacy. International Journal of Pharmaceutical Compounding Issue: Sep/Oct 1997 - Home Health Care URL: http://www.ijpc.com/Products/ProductAddToCart.cfm?PID=1 263

23. Cheryl A. Thompson; Recent FDA compounding guidances to pharmacies may not apply everywhere, American Journal of Health-System Pharmacy, Volume 75, Issue 5, 1 March 2018, Pages 258, https://doi.org/10.2146/news 180015

24. Gigi D. Veterinary Compounding: Regulation, Challenges, and Resources Pharmaceutics. 2017 Mar; 9(1): 5. doi: [10.3390/pharmaceutics9010005] PMID: 28075379

25. Schommer JC, Brown LM, Sogol EM. Section: Nuclear Pharmacy. Career Pathway Evaluation Program 2007 Pharmacist Profile Survey. June 2007.

26. SECTION. 2700 - Nuclear Pharmacy URL: http://www.ncbop.org/LawsRules/rules.2700.pdf

27. APhA Home. Nuclear Pharmacy Practice. URL: https://pharmacist.com/nuclear-pharmacy-practice

28. Lam MS. Extemporaneous compounding of oral liquid dosage formulations and alternative drug delivery methods for anticancer drugs. Pharmacother 2011;31(2):164-192. doi: 10.1592/phco.31.2.164. Review. PubMed PMID: 21275495.

29. Mulla H, Tofeig M, Bu'Lock F, Samani N, Pandya HC. Variations in captopril formulations used to treat children with heart failure: a survey in the United kingdom. Arch Dis Child 2007;92(5):409-411.

30. Special feature. Why specials manufacturing units are needed now as much as they ever were. Pharm J 2005;275:143-144.

31. Patel VP, Desai TR and others. EXTEMPORANEOUS DOSAGE FORM FOR ORAL LIQUIDS. Pharm 2011;2(2):86-103.

32. Lopez FL, Ernest TB, Tuleu C, Gul MO. Formulation approaches to pediatric oral drug delivery: benefits and limitations of current platforms. Expert Opin Drug Deliv 2015;12(11):1727-1740.

33. Catherine M. Crill, Emily B. Hak, Lawrence A. Robinson, Richard A. Helms; Evaluation of microbial contamination associated with different preparation methods for neonatal intravenous fat emulsion infusion. Am J Health-Syst Pharm 2010;67(11):914-918. https://doi.org/10.2146/ajhp090199

34. Francis J, Abraham S. Clinical pharmacists: Bridging the gap between patients and physicians. Saudi Pharm J 2014;22(6):600-602.

35. Goldman MP. Sodium tetradecyl sulfate for sclerotherapy treatment of veins: is compounding pharmacy solution safe? Dermatol Surg 2004;30(12):1454-1456. doi: 10.1097/00042728-200412010-00005.

36. Bai S, Dormer N, Shoults C. Palatability of a novel oral formulation of prednisone in healthy young adults. J Pharm Pharmacol 2017;69(4):489-496.

37. Gee SC, Hagemann TM. Palatability of liquid anti-infectives: clinician and student perceptions and practice outcomes. $J$ Pediatr Pharmacol Ther 2007;12(4):216-223.

38. Mistry P, Stirling H, Callens C, Hodson J, Batchelor H, SPaeDD-UK project. Evaluation of patient-reported outcome measurements as a reliable tool to measure acceptability of the taste of paediatric medicines in an inpatient paediatric population. BMJ Open 2018;8(7):e021961. Published 2018 Jul 11. doi:10.1136/bmjopen-2018-021961

39. Santoveña-Estévez A, Suárez-González J, Vera M, GonzálezMartín C, Soriano M, Fariña JB. Effectiveness of Antimicrobial Preservation of Extemporaneous Diluted Simple Syrup Vehicles for Pediatrics. J Pediatr Pharmacol Ther 2018;23(5):405-409. 
40. Guidance for Pharmacists on Extemporaneous Dispensing Pharmaceutical Society of Ireland Version 1 June 2015

41. Lowey A, Jackson M. How to ensure the quality and safety of unlicensed oral medicines. Pharm J 29 August, 2008

42. Terrie YC. Pharmacy Compounding Is Flourishing Once Again. Pharmacy Times ${ }^{\circledR}$ November 01, 2005

43. Erickson MA. Your Compounding Questions Answered. Pharmacy Times® September 17, 2013

How to cite this article: Mohiuddin AK. Extemporaneous compounding: Cautions, controversies and convenience. Int $\mathbf{J}$ Comprehensive Adv Pharmacol 2018;3(4):124-137. 